\title{
Statin therapy is associated with improved survival in patients with ventricular tachyarrhythmias
}

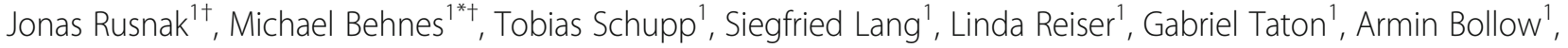 \\ Thomas Reichelt ${ }^{1}$, Dominik Ellguth' ${ }^{1}$, Niko Engelke', Uzair Ansari ${ }^{1}$, Ibrahim El-Battrawy ${ }^{1}$, Thomas Bertsch², \\ Christoph A. Nienaber ${ }^{3}$, Muharrem Akin ${ }^{4}$, Kambis Mashayekhi ${ }^{5}$, Christel Weiß ${ }^{6}$, Martin Borggrefe ${ }^{1}$ and Ibrahim Akin ${ }^{1}$
}

\begin{abstract}
Objectives: The study sought to assess the impact of statin therapy on survival in patients presenting with ventricular tachyarrhythmias.

Background: Data regarding the outcome of patients with statin therapy presenting with ventricular tachyarrhythmias is limited.

Methods: A large retrospective registry was used including all consecutive patients presenting with ventricular tachycardia (VT) or fibrillation (VF) from 2002 to 2016. Patients with statin were compared to patients without statin therapy (non-statin). The primary prognostic endpoint was long-term all-cause death at 3 years. Uni- and multivariable Cox regression analyses were applied in propensity-score matched cohorts.

Results: A total of 424 matched patients was included. The rates of VT and VF were similar in both groups $(\mathrm{VT}$ : statin $71 \%$ vs. non-statin 68\%; VF: statin 29\% vs. 32\%; $p=0.460$ ). Statin therapy was associated with lower all-cause mortality at longterm follow-up (mortality rates 16\% versus 33\%; log rank, $p=0.001 ; \mathrm{HR}=0.438 ; 95 \% \mathrm{Cl} 0.290-0.663 ; p=0.001$ ), irrespective of the underlying type of ventricular tachyarrhythmia (VTNF), left ventricular ejection fraction (LVEF) > 35\%, presence of an activated implantable cardioverter defibrillator (ICD), cardiogenic shock or cardiopulmonary resuscitation (CPR).
\end{abstract}

Conclusion: Statin therapy is independently associated with lower long-term mortality in patients presenting with ventricular tachyarrhythmias on admission.

Trial registration: Clinicaltrials.gov, NCT02982473, 11/29/2016, Retrospectively registered.

Keywords: Sudden cardiac death, Ventricular tachyarrhythmias, Ventricular fibrillation, Statin therapy

\section{Condensed abstract}

This study retrospectively examined the impact of a statin therapy on survival in 424 propensity-matched patients admitted with ventricular tachyarrhythmias. Presence of statin therapy was independently associated with lower long-term mortality (mortality rates 16\% versus 32\%; log rank $p=0.001 ; \mathrm{HR}=0.438 ; 95 \%$ CI 0.290-0.663; $p=0.001$ ).

\footnotetext{
* Correspondence: michael.behnes@umm.de

†Jonas Rusnak and Michael Behnes contributed equally to this work.

${ }^{1}$ First Department of Medicine, University Medical Centre Mannheim (UMM), Faculty of Medicine Mannheim, University of Heidelberg, European Center for AngioScience (ECAS), and DZHK (German Center for Cardiovascular Research) partner site Heidelberg/Mannheim, Mannheim, University of Heidelberg, Theodor-Kutzer-Ufer 1-3, 68167 Mannheim, Germany Full list of author information is available at the end of the article
}

\section{Introduction}

Epidemiological studies demonstrated a distinct association between cholesterol levels and all-cause as well as cardiovascular mortality [1-4]. Therefore, international guidelines recommend lipid-lowering therapy in patients at risk for and with already established cardiovascular diseases (CVD) as an effective treatment for secondary and primary prevention of atherosclerotic cardiovascular disease. Statins competitively inhibit HMG-CoA reductase activity and currently represent the first-line therapy in lowering lipid-levels $[3,5]$.

Large, randomized controlled trials (RCT) in patients with acute myocardial infarction (AMI) or coronary

C The Author(s). 2019 Open Access This article is distributed under the terms of the Creative Commons Attribution 4.0 International License (http://creativecommons.org/licenses/by/4.0/), which permits unrestricted use, distribution, and 
artery disease (CAD) demonstrated that lipid lowering therapy by statins (i.e. simvastatin or pravastatin), was associated with a reduced all-cause and cardiovascular mortality at five to six years $[6,7]$. Furthermore, statin therapy was also investigated as an adjunct therapy in patients with chronic heart failure due to any cause. However, within the RCTs, namely GISSI-HF and CORONA, the use of rosuvastatin was not associated with a reduction of mortality or sudden cardiac death (SCD) at three to four years $[8,9]$. The authors based their study concept on the multiple mechanisms of statins beyond prevention in artheroslerosis and therefore included any kind of heart failure patients without any strict inclusion criteria independently of cholesterol cutoffs for the use of rosuvastatin [10].

The multiple mechanisms of statins beyond lipid-lowering have been described recently and may include additional anti-arrhythmic effects. Statins stabilize the atherosclerotic and ischemic burden, which might indirectly translate into anti-arrhythmic effects inhibiting the onset of ventricular tachyarrhythmias and SCD. A direct antiarrhythmic effect apart from affecting arterosclerosis has also been suggested $[11,12]$. Several observational studies investigating mostly patients with heart failure showed a reduction of rates of ventricular tachycardia (VT), ventricular fibrillation (VF) and SCD, as well as reduced rates of appropriate ICD-therapy, which might in turn impact secondary reduction of mortality due to ventricular tachyarrhythmias [10, 13-20]. However, no data is available at present, whether statins may impact secondary long-term survival in patients after presenting with ventricular tachyarrhythmias on admission.

Therefore, the present study evaluates the prognostic impact of statin therapy on long-term survival in patients presenting with ventricular tachyarrhythmias on admission.

\section{Methods}

\section{Study patients, design and data collection}

The present study retrospectively included all consecutive patients presenting with ventricular tachyarrhythmias on hospital admission from 2002 until 2016 at the First Department of Medicine, University Medical Centre Mannheim, Germany. Using the hospital information system, all relevant clinical data related to the index event were documented. The data, analytic methods, and study materials will be made available to other researchers for purposes of reproducing the results or replicating the procedure onreasonable personal request to the corresponding author.

Ventricular tachyarrhythmias comprised VT and VF, as defined by current international guidelines [21]. Sustained VT was defined by duration of more than $30 \mathrm{~s}$ or causing hemodynamic collapse within $30 \mathrm{~s}$. Non-sustained VT was definded by duration of less than $30 \mathrm{~s}$ both with wide QRS complex ( $\geq 120$ milliseconds) at a rate greater than 100 beats per minute [21]. Ventricular tachyarrhythmias were documented by 12-lead ECG (electrocardiography), ECG telemonitoring, ICD or in case of unstable course or during resuscitation pulmonary resuscitation (CPR) by external defibrillator monitoring. Documented VF was treated by external defibrillation and in case of prolonged instability with additional intravenous anti-arrhythmic drugs during CPR.

Further data being documented contained baseline characteristics, prior medical history, prior medical treatment, length of index stay, detailed findings of laboratory values at baseline, data derived from all non-invasive or invasive cardiac diagnostics and device therapies, such as coronary angiography, electrophysiological examination, data being derived from prior or newly implanted cardiac devices, including those ICD already implated at index and at follow-up, pacemakers or cardiac contractility modulation $(\mathrm{CCM})$, as well as imaging modalities, such as echocardiography or cardiac magnetic resonance imaging (cMRI).

Every revisit at the outpatient clinic or re-hospitalization was documented when related to recurrent ventriculartachyarrhythmias and adverse cardiac events. Adverse cardiac events comprised acute heart failure, CPR, cardiac surgery, recurrent percutaneous coronary intervention, new implants or upgrades of cardiac devices, and worsening or improvement of left ventricular function.

Documentation period lasted from index event until 2016. Documentation of all medical data was performed by independent cardiologists at the time of the patients' individual period of clinical presentation, being blinded to final data analyses.

The present study is derived from an analysis of the "Registry of Malignant Arrhythmias and Sudden Cardiac Death - Influence of Diagnostics and Interventions (RACE-IT)" and represents a single-center registry including consecutive patients presenting with ventricular tachyarrhythmias, being acutely admitted to the University Medical Center Mannheim (UMM), Germany (clinicaltrials.gov identifier: NCT02982473) from 2002 until 2016. The registry was carried out according to the principles of the declaration of Helsinki and was approved by the medical ethics commission II of the Faculty of Medicine Mannheim, University of Heidelberg, Germany.

The medical center covers a general emergency department for emergency admission of traumatic, surgical, neurological and cardiovascular conditions. Interdisciplinary consultation is an inbuilt feature of this $24 / 7$ service, and connects to a stroke unit, four intensive care units (ICU) with extracorporeal life support and a chest pain unit (CPU) to alleviate rapid triage of patients. The cardiologic department itself includes a $24 \mathrm{~h}$ catheterization laboratory, an electrophysiologic laboratory, a hybrid operating room and telemetry units. 
Index events, risk stratification measures and prognostic outcome

For the present study, all patients surviving index hospitalization after presenting with ventricular tachyarrhythmias between 2002 and 2016 were included. Each patient was counted only once for inclusion when presenting with the first episode of ventricular tachyarrhythmias. Indication to treat patients with statins was based on European guidelines of statin therapy [3]. Overall exclusion criteria comprised patients without complete follow-up data regarding mortality.

The primary prognostic endpoint was all-cause mortality during the follow-up period until 2016. All-cause mortality was documented using our electronic hospital information system and by directly contacting state resident registration offices ("bureau of mortality statistics") across Germany. Identification of patients was verified by name, surname, day of birth and registered living address. In 48 patients, no data on patients' survival could have been retrieved, as those patients were not even reachable by telephone, and were therefore excluded from final analyses (corresponding lost to follow-up rate of 1.7\%).

\section{Statistical methods}

Quantitative data are presented as mean \pm standard error of mean (SEM), median and interquartile range (IQR), and ranges depending on the distribution of the data and were compared using the Student's $t$ test for normally distributed data or the Mann-Whitney $U$ test for nonparametric data. Deviations from a Gaussian distribution were tested by the Kolmogorov-Smirnov test. Spearman's rank correlation for nonparametric data was used to test univariate correlations. Qualitative data are presented as absolute and relative frequencies and compared using the $\mathrm{Chi}^{2}$ test or the Fisher's exact test, as appropriate.

The following analyses were applied stepwise to evaluate the prognostic impact of statin therapy on all-cause mortality:

Propensity score analyses were performed, since this study includes consecutively all patients with ventricular tachyarrhythmias without randomization [22, 23]. Accordingly, propensity scores (probability for belonging to statin = yes) were calculated for each individual based predefined variables (see below). Afterwards, matched pairs were created using the method of nearest neighbor matching with a caliper distance of $5 \%$. This means: each pair consisted of one individual with statin $=$ yes and statin $=$ no, whose propensity scores differed by less than $5 \%$. We found 212 pairs with mean propensity score $0.5931+/-0.3113$ (statin therapy $=0$ ) and $0.6065+/-0.3121$ (statin therapy $=1$ ).

Uni-variable stratification was performed using the Kaplan-Meier method with comparisons between groups using uni-variable hazard ratios (HR) given together with 95\% confidence intervals, according to the presence of a statin therapy within the propensity-matched cohorts.

Multivariable Cox regression models were developed using the "forward selection" option, where only statistically significant variables $(p<0.05)$ were included and analyzed simultaneously (see below). Multivariable Cox regressions were applied in the propensity-matched cohorts.

Predefined variables being used for propensity score matching (step A) and multivariable Cox-regressions (step C) included: baseline parameters (age, gender), chronic diseases (diabetes, chronic kidney disease (glomerular filtration rate $<90 \mathrm{~mL} / \mathrm{min} / 1.73 \mathrm{~m}^{2}$ ), left ventricular dysfunction), acute comorbidities (cardiogenic shock, cardiopulmonary resuscitation (CPR), acute myocardial infarction), presence of an implanted cardiac defibrillator (ICD), and underlying ventricular tachyarrhythmia (i.e. $\mathrm{VT} / \mathrm{VF}$ ) on admission.

Follow-up periods for evaluation of all-cause mortality were set at 3 years (=long-term), according to the median survival of statin patients to guarantee complete survival of at least $50 \%$ of patients. Patients not meeting long-term follow-up were censored.

The result of a statistical test was considered significant for $p<0.05$, and a statistical trend for $p<0.10$. SAS, release 9.4 (SAS Institute Inc., Cary, NC, USA) and SPSS (Version 25, IBM, Armonk, New York) were used for statistics.

\section{Results}

\section{Study population}

The propensity-matched cohort of consecutive patients surviving ventricular tachyarrhythmias on admission at our institution consisted of a total of 424 patients, of which each half was treated either with or without statins. The cohort was well matched for age, gender, arterial hypertension and diabetes. Statin patients revealed a higher rate of hyperlipidaemia, smoking and prior history of myocardial infarction. Overall, most patients presented with VT compared to VF with equally distributed rates in each group (VT: 71\% versus 68\%; VF: 29\% versus $32 \%$ ) (Table 1).

Target dosages were reached already at discharge, including simvastatin as the most frequently administered statin ( $n=105 ; 50 \%$; mean dosage $29 \mathrm{mg}$ per day) followed by atorvastatin ( $n=86 ; 41 \%$; mean dosage 40 mg per day), fluvastatin ( $\mathrm{n}=10 ; 5 \%$ mean dosage $56 \mathrm{mg}$ per day) and pravastatin $(n=8 ; 4 \%$; mean dosage $21 \mathrm{mg}$ per day) (data not shown).

Notably, no differences were found in both groups regarding prognosis-relevant comorbidities, including AMI, cardiogenic shock, cardiomyopathy, PEA (pulseless electrical activity) or asystole. Statin patients suffered 
Table 1 Baseline characteristics and comorbidities after propensity score matching

\begin{tabular}{|c|c|c|c|}
\hline Characteristic & $\begin{array}{l}\text { Non statin } \\
(n=212 ; 50 \%)\end{array}$ & $\begin{array}{l}\text { statin } \\
(n=212 ; 50 \%)\end{array}$ & $p$ value \\
\hline Age, median (range) & $69(16-92)$ & $68(25-86)$ & 0.669 \\
\hline \multicolumn{4}{|l|}{ Gender, n (\%) } \\
\hline Male & (75) & (77) & 0.569 \\
\hline
\end{tabular}

Ventricular tachyarrhythmias, n (\%)

$\begin{array}{llllll}\text { Ventricular tachycardia } & 144 & (68) & 151 & (71) & 0.460 \\ \text { Ventricular fibrillation } & 68 & (32) & 61 & (29) & \\ \text { Prior medical history, } \mathrm{n}(\%) & & & & & \\ \text { Chronic heart failure } & 71 & (34) & 72 & (34) & 0.918 \\ \text { Coronary artery disease } & 102 & (48) & 102 & (48) & 1.000 \\ \text { Myocardial infarction } & 49 & (23) & 67 & (32) & 0.050 \\ \text { Prior PCl } & 41 & (19) & 58 & (27) & 0.051 \\ \text { Prior CABG } & 25 & (12) & 43 & (20) & 0.017 \\ \text { Inflammatory heart disease } & 2 & (0.9) & 3 & (1) & 1.000 \\ \text { Valvular heart disease } & 30 & (14) & 21 & (10) & 0.179 \\ \text { Implanted cardiac devices } & & & & & \\ \quad \text { ICD } & 40 & (19) & 38 & (18) & 0.802 \\ \text { Pacemaker } & 10 & (5) & 9 & (4) & 0.814 \\ \quad \text { Ablation therapy } & 10 & (5) & 4 & (2) & 0.103 \\ \text { Stroke } & 23 & (11) & 25 & (12) & 0.759 \\ \text { Chronic kidney disease } & 106 & (50) & 103 & (49) & 0.771 \\ \text { Liver cirrhosis } & 3 & (1) & 1 & (0.5) & 0.623 \\ \text { COPD/asthma } & 29 & (14) & 21 & (10) & 0.228\end{array}$

Cardiovascular risk factors, $\mathrm{n}(\%)$

Arterial hypertension $\quad 124$

Diabetes mellitus

Hyperlipidemia

Smoking

Cardiac family history

Comorbidities at index stay, $\mathrm{n}(\%)$

$\begin{array}{llllll}\text { Acute myocardial infarction } & 25 & (12) & 35 & (17) & 0.164 \\ \text { STEMI } & 3 & (1) & 9 & (4) & 0.079 \\ \text { NSTEMI } & 22 & (10) & 26 & (12) & 0.540 \\ \text { Cardiogenic shock } & 21 & (10) & 18 & (9) & 0.614 \\ \text { Atrioventricular block } & 8 & (4) & 5 & (2) & 0.398 \\ \text { Cardiomyopathy } & 36 & (17) & 36 & (17) & 1.000 \\ \text { Hyperkalemia } & 1 & (0.5) & 2 & (0.9) & 1.000 \\ \text { Hypokalemia } & 18 & (9) & 16 & (8) & 0.721 \\ \text { Stroke } & 9 & (4) & 8 & (4) & 0.804 \\ \text { Intracranial hemorrhage } & 0 & (0) & 1 & (0.5) & 1.000 \\ \text { Clinically significant bleeding } & 11 & (5) & 1 & (0.5) & \mathbf{0 . 0 0 3} \\ \text { Anemia } & 20 & (9) & 6 & (3) & \mathbf{0 . 0 0 5} \\ \text { Septic shock } & 5 & (2) & 0 & (0) & 0.061 \\ \text { Cardiac surgery } & 4 & (2) & 7 & (3) & 0.359\end{array}$

Table 1 Baseline characteristics and comorbidities after propensity score matching (Continued)

\begin{tabular}{llllll}
\hline Characteristic & \multicolumn{2}{l}{ Non statin } & \multicolumn{2}{l}{ statin } & $p$ value \\
& $(n=212 ; 50 \%)$ & \multicolumn{2}{l}{$(n=212 ; 50 \%)$} & \\
\hline Inadequate ICD shock & 2 & $(0.9)$ & 2 & $(0.9)$ & 1.000 \\
Atrial fibrillation & 84 & $(40)$ & 70 & $(33)$ & 0.157 \\
Paroxysmal & 55 & $(26)$ & 43 & $(20)$ & 0.135 \\
$\quad$ Persistent & 9 & $(4)$ & 3 & $(1)$ & \\
$\quad$ Permanent & 20 & $(9)$ & 24 & $(11)$ & \\
Asystole & 5 & $(2)$ & 9 & $(4)$ & 0.277 \\
PEA & 2 & $(0.9)$ & 0 & $(0)$ & 0.499 \\
\hline
\end{tabular}

CABG, coronary artery bypass grafting; COPD, chronic obstructive pulmonary disease; ICD, implantable cardioverter- defibrillator; NSTEMI, non-ST segment elevation myocardial infarction; PCl, percutaneous coronary intervention; PEA, pulseless electrical activity; STEMI, ST segment myocardial infarction. Bold type indicates $p<0.05$

less often from clinical significant bleeding and anaemia (Table 1).

\section{Cardiac diagnostics and therapies}

As shown in Table 2, notably no differences were found in both groups regarding LVEF, rates of CPR, external defibrillation, fibrinolysis and TTM (targeted temperature management). Additionally, no differences were observed regarding extend of $\mathrm{CAD}$ and $\mathrm{PCI}$ rates, rates of electrophysiological testing and device therapy. CABG (coronary artery bypass graft) was more common in statin patients, alongside with higher rates of treatment with beta-blocker, ACEi (angiotensin-converting-enzyme inhibitor), ASA (acetylsalicylic acid), and dual antiplatelet therapy (Table 2).

\section{All-cause mortality and survival data}

At long-term follow up (median 3.0 years (IQR 638 days - 2869 days), statin patients had significantly better survival compared to non-statin patients (long-term mortality rates $16 \%$ versus $33 \%$; $\log$ rank $p=0.001$; HR $=0.438 ;$ 95\% CI 0.290-0.663; $p=0.001$ ); (Fig. 1, left panel). Focusing on the presence of ventricular tachyarrhythmias, the prognostic benefit of statin patients was irrespective of the presence of VT (mortality rates 15\% versus $33 \%$; $\log$ rank $p=0.001 ; \mathrm{HR}=0.439 ; 95 \% \mathrm{CI}$ $0.267-0.723 ; p=0.001$ ) (Fig. 1, middle panel) or VF (mortality rates $16 \%$ versus $34 \%$; $\log$ rank $p=0.028$; HR $=0.445 ; 95 \%$ CI 0.212-0.935; $p=0.032$ ) (Fig. 1, right panel). Accordingly, long-term survival was not statistically different in statin patients presenting with VF compared to VT (mortality rates 16\% versus 15\%; log rank $p=0.796$ ) (data not shown).

The prognostic benefit of statin patients was still evident when stratifying according to left ventricular ejection fraction (LVEF) above or below 35\% (mortality rates: $\mathrm{LVEF} \geq 35,11 \%$ vs $32 \%$, log-rank $p=0.001, \mathrm{HR}=$ 
Table 2 Cardiac diagnostics, therapies and survival data after propensity score matching

\begin{tabular}{|c|c|c|c|c|c|}
\hline \multirow{2}{*}{$\begin{array}{l}\text { Characteristic } \\
\text { Left ventricular ejection function, n (\%) }\end{array}$} & \multicolumn{2}{|c|}{$\begin{array}{l}\text { No statin } \\
(n=212 ; 50 \%)\end{array}$} & \multicolumn{2}{|c|}{$\begin{array}{l}\text { statin } \\
(n=212 ; 50 \%)\end{array}$} & \multirow[t]{2}{*}{$p$ value } \\
\hline & & & & & \\
\hline LVEF $\geq 55 \%$ & 63 & $(30)$ & 60 & (28) & 0.352 \\
\hline LVEF 54-35\% & 63 & $(30)$ & 62 & (29) & \\
\hline LVEF $<35 \%$ & 86 & $(41)$ & 90 & (43) & \\
\hline \multicolumn{6}{|l|}{ Cardiac therapies at index, n (\%) } \\
\hline Cardiopulmonary resuscitation & 61 & $(29)$ & 51 & (24) & 0.271 \\
\hline In hospital & 26 & $(12)$ & 21 & (10) & 0.439 \\
\hline Out of hospital & 35 & $(17)$ & 30 & (14) & 0.500 \\
\hline External defibrillation & 59 & $(28)$ & 51 & (24) & 0.375 \\
\hline External cardioversion & 13 & (6) & 9 & (4) & 0.381 \\
\hline Systemic thrombolysis & 7 & (3) & 5 & (2) & 0.558 \\
\hline Targeted temperature management (TTM) & 5 & $(2)$ & 8 & (4) & 0.398 \\
\hline \multicolumn{6}{|l|}{ Coronary artery disease, n (\%) } \\
\hline Coronary angiography, overall & 131 & $(62)$ & 140 & (66) & 0.363 \\
\hline \multicolumn{6}{|l|}{ Coronary artery disease, n (\%) } \\
\hline No evidence of CAD & 47 & $(36)$ & 41 & (29) & 0.425 \\
\hline 1-vessel & 24 & $(18)$ & 33 & (24) & \\
\hline 2-vessel & 27 & $(21)$ & 24 & (17) & \\
\hline 3-vessel & 33 & $(25)$ & 42 & (30) & \\
\hline СТO & 32 & $(24)$ & 27 & (19) & 0.305 \\
\hline Presence of $\mathrm{CABG}$ & 15 & $(12)$ & 30 & (21) & 0.027 \\
\hline Intracoronary thrombus & 2 & $(2)$ & 6 & (4) & 0.180 \\
\hline CPR during coronary angiography & 4 & (3) & 3 & (2) & 0.715 \\
\hline $\mathrm{PCl}, \mathrm{n}(\%)$ & 28 & $(21)$ & 43 & (31) & 0.081 \\
\hline \multicolumn{6}{|l|}{ Target lesions } \\
\hline $\mathrm{RCA}$ & 11 & (5) & 16 & (8) & 0.320 \\
\hline LMT & 1 & $(0.5)$ & 0 & (0) & 1.000 \\
\hline LAD & 15 & (7) & 18 & (9) & 0.587 \\
\hline RIM & 0 & $(0)$ & 0 & $(0)$ & - \\
\hline LCX & 6 & (3) & 12 & (6) & 0.148 \\
\hline Bypass graft & 0 & (0) & 3 & (1) & 0.248 \\
\hline \multicolumn{6}{|l|}{ Electrical therapies at index, n (\%) } \\
\hline Electrophysiological examination & 72 & (34) & 88 & $(42)$ & 0.109 \\
\hline Catheter ablation & 18 & (9) & 19 & (9) & 0.863 \\
\hline \multicolumn{6}{|l|}{ Newly implanted devices at index, n (\%) } \\
\hline ICD & 54 & $(26)$ & 54 & (26) & 1.000 \\
\hline CRT-D & 3 & (1) & 8 & (4) & 0.127 \\
\hline CRT-P & 1 & $(0.5)$ & 0 & (0) & 1.000 \\
\hline Pacemaker & 4 & $(2)$ & 2 & $(0.9)$ & 0.685 \\
\hline Cardiac contractility modulation (CCM) & 0 & $(0)$ & 0 & (0) & - \\
\hline Subcutaneous ICD & 0 & $(0)$ & 1 & $(0.9)$ & 1.000 \\
\hline Overall implanted devices & 124 & $(59)$ & 121 & $(57)$ & 0.768 \\
\hline
\end{tabular}

Medication at discharge, $\mathrm{n}(\%)$ 
Table 2 Cardiac diagnostics, therapies and survival data after propensity score matching (Continued)

\begin{tabular}{|c|c|c|c|c|c|}
\hline \multirow{2}{*}{$\begin{array}{l}\text { Characteristic } \\
\text { Beta-blocker }\end{array}$} & \multicolumn{2}{|c|}{$\begin{array}{l}\text { No statin } \\
(n=212 ; 50 \%)\end{array}$} & \multicolumn{2}{|c|}{$\begin{array}{l}\text { statin } \\
(n=212 ; 50 \%)\end{array}$} & \multirow{2}{*}{$\begin{array}{l}p \text { value } \\
\mathbf{0 . 0 0 1}\end{array}$} \\
\hline & 162 & (76) & 187 & (88) & \\
\hline ACE-inhibitor & 115 & (54) & 153 & (72) & 0.001 \\
\hline AT1-Antagonist & 21 & (10) & 29 & (14) & 0.249 \\
\hline Aldosteron-antagonist & 22 & (10) & 33 & (16) & 0.112 \\
\hline Aspirin only & 58 & (27) & 78 & (37) & 0.037 \\
\hline Thienopyridine only & 4 & (2) & 10 & (5) & 0.103 \\
\hline Dual antiplatelet therapy & 25 & (12) & 52 & (25) & 0.001 \\
\hline Vitamin $\mathrm{k}$ antagonist & 54 & (26) & 48 & (23) & 0.495 \\
\hline NOAC & 7 & (3) & 2 & $(0.9)$ & 0.175 \\
\hline Amiodarone & 54 & (26) & 38 & (18) & 0.059 \\
\hline Digitalis & 33 & (16) & 38 & (18) & 0.515 \\
\hline \multicolumn{6}{|l|}{ Hospitalization time, days, (median (IQR)) } \\
\hline Total hospitalization time & \multicolumn{2}{|c|}{$16(8-27)$} & \multicolumn{2}{|c|}{$14(8-26)$} & 0.009 \\
\hline ICU time & \multicolumn{2}{|c|}{$3(0-11)$} & \multicolumn{2}{|c|}{$2(0-7)$} & 0.004 \\
\hline Follow-up time, days, (mean; median (range)) & \multicolumn{2}{|c|}{$\begin{array}{l}1849 ; 1806 \\
(25-5095)\end{array}$} & \multicolumn{2}{|c|}{$\begin{array}{l}1853 ; 1675 \\
(18-5091)\end{array}$} & 0.013 \\
\hline All cause-mortality at 3 years, $n(\%)$ & 70 & (33) & 33 & (16) & 0.001 \\
\hline
\end{tabular}

CABG; coronary artery bypass grafting; CAD, coronary artery disease; CRT-D, cardiac resynchronization therapy plus defibrillator; CRT-P, cardiac resynchronization therapy plus pacemaker; CTO, chronic total occlusion; LAD, left anterior descending; LCX, left circumflex; LVEF, left ventricular ejection function; ICD, implantable cardioverter-defibrillator; ICU, intensive care unit; IQR, interquartile range; NOAC, new oral anticoagulants; PCl, percutaneous coronary intervention; RCA, right coronary artery; RIM, Ramus intermedius. Bold type indicates $p<0.05$

$0.302,95 \%$ CI $=0.162-0.565, p=0.001 ;$ LVEF $<35,21 \%$ vs $35 \%$; log rank $p=0.089$ ) (Fig. 2, left and right panel).

The presence of an activated ICD was associated with a comparable lower subsequent mortality both in statin (mortality rates: statin patients, $12 \%$ vs $21 \%$, log rank $p$ $=0.040, \mathrm{HR}=0.493,95 \% \mathrm{CI}=0.247-0.2983, p=0.045$ ) and non-statin patients (mortality rates: non-statin patients, $25 \%$ vs $44 \%$, log rank $p=0.002, \mathrm{HR}=0.490,95 \%$ $\mathrm{CI}=0.305-0.785, p=0.003$ ) (data not shown). Furthermore, irrespective of the presence or absence of activated ICD mortality was still lower in statin compared to non-statin patients (mortality rates: ICD carriers, $12 \%$ vs $25 \%$, log-rank $p=0.007, \mathrm{HR}=0.429,95 \% \mathrm{CI}=0.227-$
$0.804, p=0.008$; no ICD carrier, $21 \%$ vs $44 \%$, log-rank $\mathrm{p}=0.002, \mathrm{HR}=0.439,95 \% \mathrm{CI}=0.252-0.754, \mathrm{p}=0.003)$ (Fig. 3, left \& right panel).

\section{Multivariable cox models}

In multivariable Cox regression analysis, statin therapy was still associated with a lower risk of all-cause mortality in patients presenting with ventricular tachyarrhythmias (HR 0.508; CI 0.333-0.776, $p=0.002$ ), comparable to the presence of an activated ICD (HR 0.359, 95\% CI 0.359; 95\% CI $0.230-0.559 ; p=0.002$ ). Furthermore, patients' age (HR 1.054), male gender (HR 1.382), CKD (HR 1.356), LVEF <35\% (HR 1.803) and cardiogenic

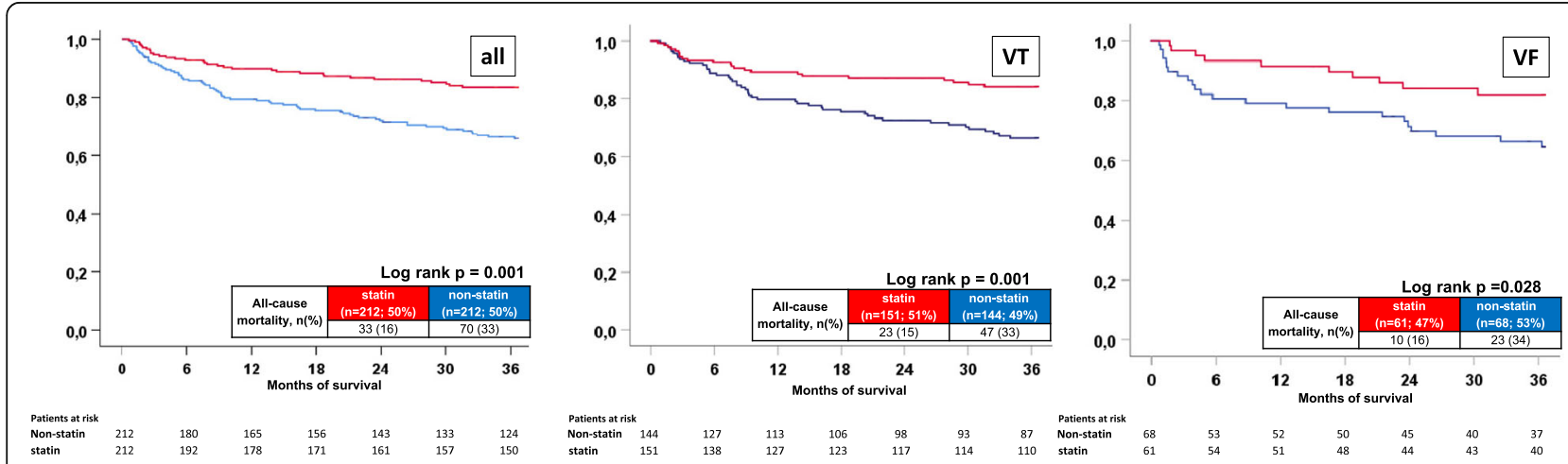

Fig. 1 Overall all-cause mortality comparing statin with non statin patients (first panel), according to the underlying ventricular tachyarrhythmias, VT (second panel) and VF (third panel) 

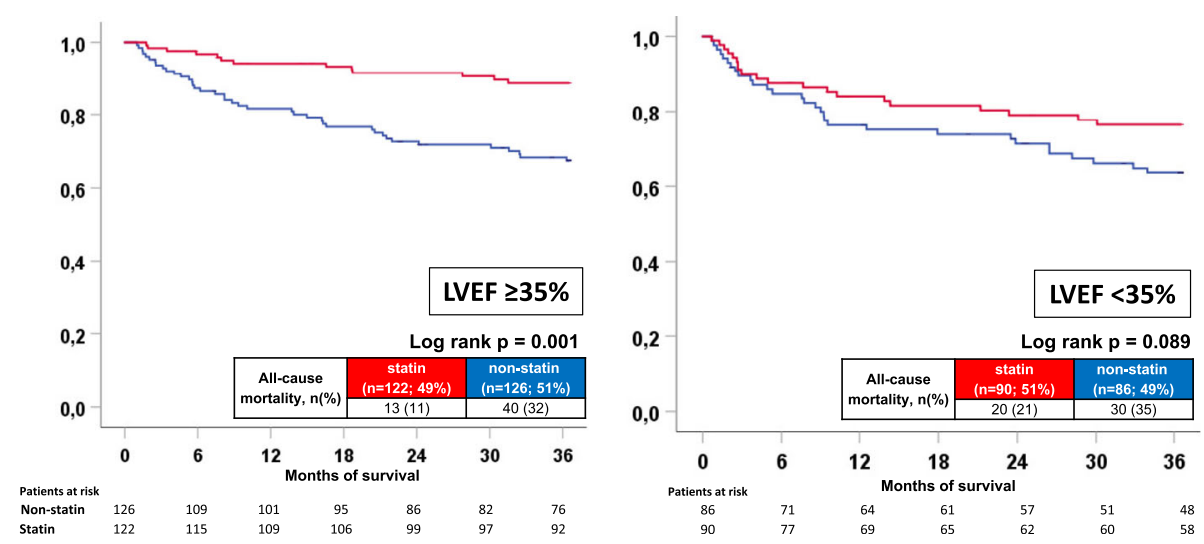

Fig. 2 All-cause mortality comparing statin with non-statin patients according to LVEF $\geq 35 \%$ (left) and LVEF < 35\% (right)

shock (HR 1.512) were significantly associated with an increased risk of long-term all-cause death. (Fig. 4).

\section{Discussion}

The present study evaluates the prognostic impact of statin therapy on long-term survival in high-risk patients surviving ventricular tachyarrhythmias on admission.

This real-world data suggests that the presence of statin therapy is associated with a reduced risk of long-term all-cause mortality. The prognostic benefit of a statin therapy was evident irrespective of the underlying type of ventricular tachyarrhythmias (VT or VF), LV dysfunction or presence of an activated ICD. Additionally, the prognostic benefit associated with statin therapy was still evident even after controlling for patients' age, gender, diabetes, CKD, AMI, CAD, cardiogenic shock, CPR, LVEF <35\% and presence of an activated ICD.

This study consistently identifies the presence of a statin therapy as a robust predictor of improved survival in patients surviving malignant arrhythmia on hospital admission. The major strength of the present study consists in the consecutive recruitment of patients with ventricular tachyarrhythmias and documented statin therapy straight from the admission scenario.

Several RCT and observational studies were able to demonstrate that lipid lowering therapy, especially by the use of statins, might lower all-cause mortality and cardiovascular death in patients with ischemic cardiomyopathy or CAD $[6,7,14,15,18]$. Statins may also prevent future $\mathrm{SCD}$, reduce recurrences of ventricular tachyarrhythmias and improve overall survival in patients suffering from heart failure patients with $\operatorname{LVEF}<30 \%[10,15]$. Notably, there is only one RCT published by Vrtovec et al. demonstrating that treatment with atorvastatin was able to significantly reduce the incidence of future SCD in patients with LVEF $<30 \%$ and cholesterol levels $>150 \mathrm{mg} / \mathrm{dl}$ at one year of follow-up [24]. Furthermore, several observational sub-studies of randomized ICD/CRT trials (MADIT II, MADIT-CRT, DEFINITE) demonstrated improved survival and reduction of arrhythmic deaths in patients treated with statins $[10,18,19]$. For instance, a sub-study of the MADIT-CRT trial demonstrated a significant reduction of appropriate ICD-therapies at 4 years in patients suffering from both ischemic and

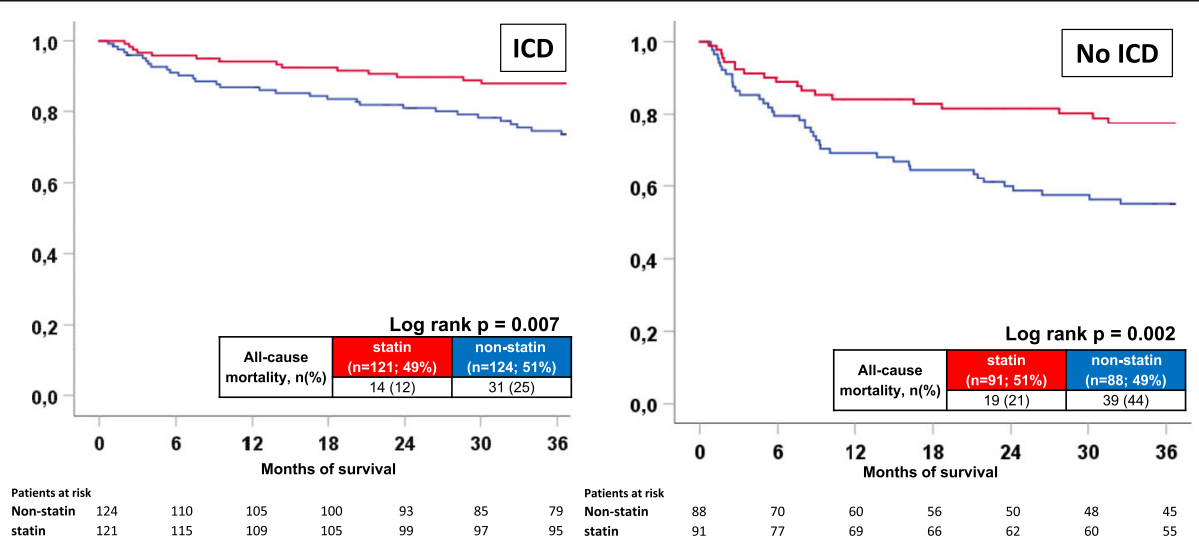

Fig. 3 All-cause mortality comparing statin with non-statin patients according to the presence (left) or absence of activated ICD (right) 
Matched cohort $(n=424)$

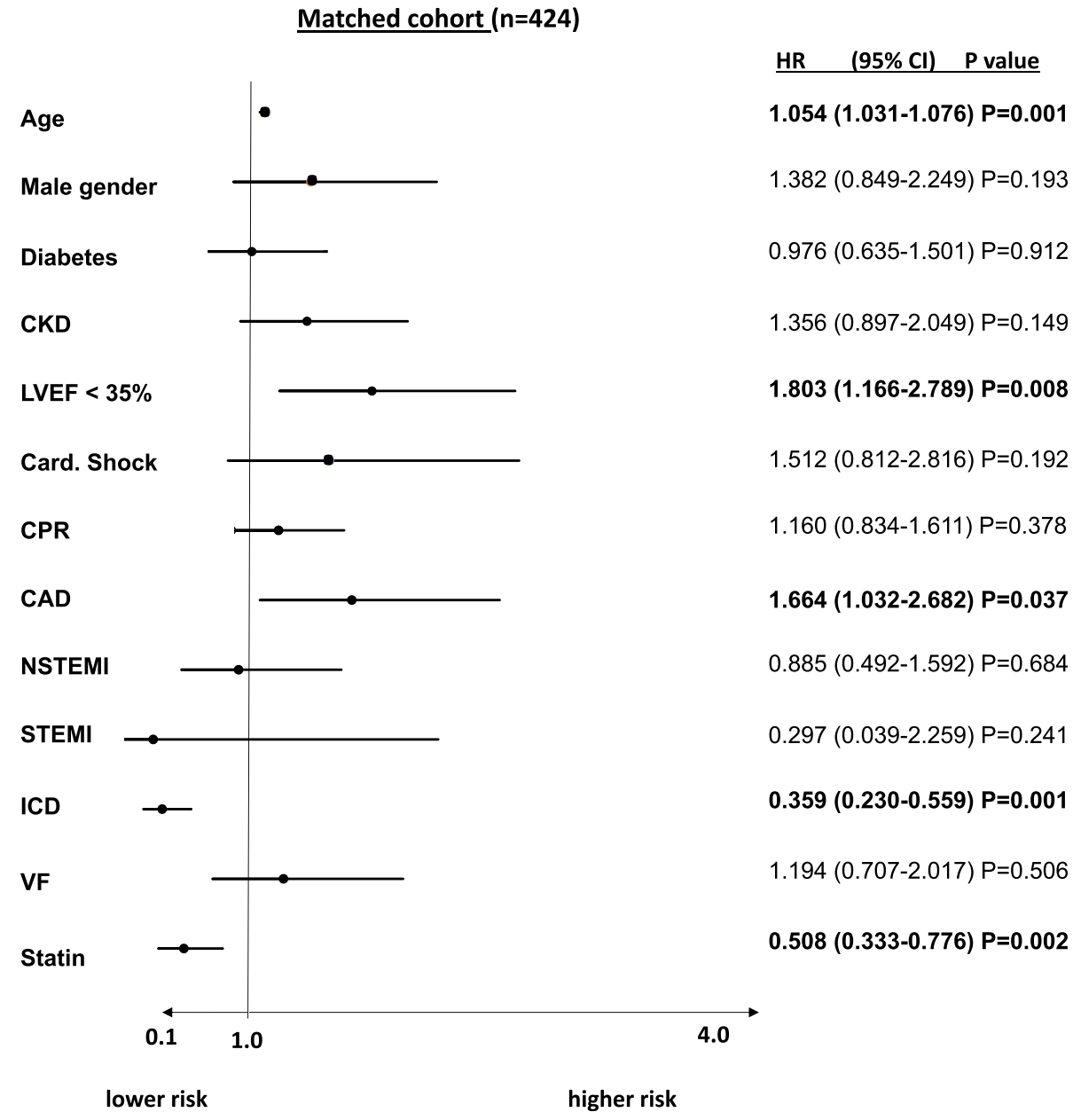

Fig. 4 Statin therapy was still associated with beneficial survival even after adjusting for several prognosis-relevant factors

non-ischemic cardiomyopathy in the presence of statin therapy. Unfortunately, no information was given on the types and dosages of the administered statins [10]. Another sub-study of the DEFINITE trial found a reduced rate of premature beats, non-sustained VT and arrhythmic deaths in ICD carriers with non-ischemic cardiomyopathy with statin therapy [19]. However, the major limitation of these sub-studies is related to their preselected study cohorts and their unmatched character regarding the use of statins, which finally lead to further conflicting results in the according meta-analyses $[12,13,17]$.

The following pathophysiological concepts may explain the beneficial effects of statin therapy in ventricular tachyarrhythmias and SCD, which may lay beyond the sole reduction of the atherosclerotic or ischemic burden. Statins are inhibitors of the HMG-CoA reductase and do reveal further pleiotropic pharmacological effects. These comprise prevention of ischemia, primary antiarrhythmic and anti-inflammatory effects [25]. The prevention of ischemia by statins was recently attributed to anti-coagulant and anti-thrombogenic effects, which in turn may reduce coronary micro-embolisms potentially causing myocardial ischemia and alleviating the development of ventricular tachyarrhythmias and SCD [25-28]. The antiinflammatory effects of statins are represented mainly by their direct influence on atherosclerosis development. Inflammation itself represents a relevant causal factor for arrhythmogenesis [25, 29]. Within a community-based RCT including 1702 patients without evidence of CVD, statins were shown to reduce elevated CRP levels (C-reactive protein), which may indirectly lead to reduced risk of SCD [30, 31]. Furthermore, statins reveal direct anti-arrhythmic effects [32]. The antiarrhythmic properties were observed in experimental mouse models, where statins attenuated $\mathrm{T}$-wave and $\mathrm{Ca} 2+$ alternans in isolated ventricular cardiomyocytes of mice treated with statin [33]. Both electrical disorders are precursors for an unstable electrical milieu alleviating the onset of malignant ventricular arrhythmia [23-25].

The use of statins is usually investigated in clinical studies investigating preselected patient populations, such as CAD, AMI, chronic heart failure or hyperlipidaemia, 
which sets the present study in clear contrast to the currently available evidence. The present study is based on an unselected all-comers design, thereby including "real-world" patients consecutively presenting on admission with life-threatening ventricular tachyarrhythmias, and still demonstrated beneficial effects for prognosis associated with statin therapy in this "high-risk" cohort. The findings were consistently and robustly demonstrated by applying stepwise stratification within a propensity score matched analysis. Underlying pathologies were strictly matched for included AMI (14\%), CAD (68\%), cardiogenic shock (10\%), cardiomyopathy (17\%) and atrial fibrillation (36\%).

There is only one study by De Sutter et al., which is widely comparable to the data of the present study. Here, CAD patients presenting at index with life-threatening ventricular tachyarrhythmias were included following secondary implantation of an ICD with a mean follow-up of 490 days [34]. Only those patients with lipid lowering therapy (defined as treatment with both statins and fibrates) revealed less recurrences of ventricular tachyarrhythmias as documented by application of anti-tachycardia pacing (ATP) or shocks. The study did not exclude patients with normal or mildly reduced LVEF. Accordingly, the present study delivers evidence that statins may reveal beneficial effects also in high-risk patients with ventricular tachyarrhythmias with a still preserved left ventricular ejection fraction (LVEF $>55,30 \%$ in each matched group).

Therefore, the present results support the hypothesis that statins may reveal several pleiotropic effects including potential anti-arrhythmic properties, since a significant reduction of secondary all-cause mortality was demonstrated in unselected but well-matched all-comer patients presenting with ventricular tachyarrhythmias on hospital admission when treated with statins.

\section{Study limitations}

This observational and retrospective registry-based analysis reflects a realistic picture of consecutive health-care supply of high-risk patients presenting with ventricular tachyarrhythmias. Lost to follow-up rate regarding the evaluated endpoint of all-cause mortality was minimal. Additionally, heterogeneity within the study population was controlled by a stepwise statistical approach including multivariable adjustment for several important comorbidities and risk factors within a propensity matched cohort. Patients not surviving out of hospital CPR and not being transferred to the heart centre were not included in this study. All clinical data was documented reliably by individual cardiologists and specialists in internal medicine and cardiology during routine clinical care being blinded to final data analyses, alleviating the use of an independent clinical event committee.

\section{Conclusions}

Statin therapy is associated with decreased long-term all-cause mortality in patients presenting with ventricular tachyarrhythmias.

\section{Acknowledgements \\ Not applicable.}

\section{Funding}

This research received no specific grant from any funding agency in the public, commercial or not-for-profit sectors.

\section{Availability of data and materials}

The datasets during and/or analyzed during the current study are available from the corresponding author on reasonable request.

\section{Authors' contributions}

JR: substantially contributed to the conception and design of the work, data acquisition and analysis as well as interpretation of data for the work, and drafted the work and revisited for critically important intellectual content. MBe: substantially contributed to the conception and design of the work, data acquisition and analysis as well as interpretation of data for the work, and drafted the work and revisited for critically important intellectual content. TS: substantially contributed to data acquisition and analysis as well as interpretation of data for the work and revisited for critically important intellectual content. SL: substantially contributed to data acquisition and analysis as well as interpretation of data for the work and revisited for critically important intellectual content. LR: substantially contributed to data acquisition and analysis as well as interpretation of data for the work and revisited for critically important intellectual content. GT: substantially contributed to data acquisition and analysis as well as interpretation of data for the work and revisited for critically important intellectual content. AB: substantially contributed to data acquisition and analysis as well as interpretation of data for the work and revisited for critically important intellectual content. TR: substantially contributed to data acquisition and analysis as well as interpretation of data for the work and revisited for critically important intellectual content. DE: substantially contributed to data acquisition and analysis as well as interpretation of data for the work and revisited for critically important intellectual content. NE: substantially contributed to data acquisition and analysis as well as interpretation of data for the work and revisited for critically important intellectual content. UA: substantially contributed to data acquisition and analysis as well as interpretation of data for the work and revisited for critically important intellectual content. IE-B: substantially contributed to data acquisition and analysis as well as interpretation of data for the work and revisited for critically important intellectual content. TB: substantially contributed to data acquisition and analysis as well as interpretation of data for the work and revisited for critically important intellectual content. CN: substantially contributed to data acquisition and analysis as well as interpretation of data for the work and revisited for critically important intellectual content. MA: substantially contributed to data acquisition and analysis as well as interpretation of data for the work and revisited for critically important intellectual content.

KM: substantially contributed to data acquisition and analysis as well as interpretation of data for the work and revisited for critically important intellectual content. CW: substantially contributed to data acquisition and analysis as well as interpretation of data for the work and revisited for critically important intellectual content. MBo: substantially contributed to conception and design of the work and revisited for critically important intellectual content. IA: conceived the study, substantially contributed to the conception and design of the work, data acquisition and analysis as well as interpretation of data for the work, and drafted the work and revisited for critically important intellectual content. All authors read and approved the final manuscript.

\section{Ethics approval and consent to participate}

The registry was carried out according to the principles of the declaration of Helsinki and was approved by the medical ethics committee II of the Medical Faculty Mannheim, University of Heidelberg, Germany. 


\section{Competing interests}

The authors declare that they have no competing interests.

\section{Publisher's Note}

Springer Nature remains neutral with regard to jurisdictional claims in published maps and institutional affiliations.

\section{Author details}

'First Department of Medicine, University Medical Centre Mannheim (UMM), Faculty of Medicine Mannheim, University of Heidelberg, European Center for AngioScience (ECAS), and DZHK (German Center for Cardiovascular Research) partner site Heidelberg/Mannheim, Mannheim, University of Heidelberg, Theodor-Kutzer-Ufer 1-3, 68167 Mannheim, Germany. Institute of Clinical Chemistry, Laboratory Medicine and Transfusion Medicine, General Hospital Nuremberg, Paracelsus Medical University, Nuremberg, Germany. ${ }^{3}$ Royal Brompton and Harefield Hospitals, NHS, London, UK. ${ }^{4}$ Department of Cardiology and Angiology, Hannover Medical School, Hannover, Germany. ${ }^{5}$ Clinic for Cardiology and Angiology II, Universitaetszentrum Freiburg Bad Krozingen, University of Freiburg, Bad Krozingen, Germany. ${ }^{6}$ Institute of Biomathematics and Medical Statistics, University Medical Center Mannheim (UMM), Faculty of Medicine Mannheim, Heidelberg University, Mannheim, Germany.

\section{Received: 16 May 2018 Accepted: 8 March 2019}

Published online: 24 May 2019

\section{References}

1. Unal B, Sozmen K, Arik H, Gerceklioglu G, Altun DU, Simsek H, Doganay S, Demiral Y, Aslan O, Bennett K, et al. Explaining the decline in coronary heart disease mortality in Turkey between 1995 and 2008. BMC Public Health. 2013;13:1135.

2. Bjorck L, Rosengren A, Bennett K, Lappas G, Capewell S. Modelling the decreasing coronary heart disease mortality in Sweden between 1986 and 2002. Eur Heart J. 2009:30:1046-56.

3. Catapano AL, Graham I, De Backer G, Wiklund O, Chapman MJ, Drexel H, Hoes AW, Jennings CS, Landmesser U, Pedersen TR, et al. 2016 ESC/EAS guidelines for the Management of Dyslipidaemias. Eur Heart J. 2016;37: 2999-3058.

4. Marchioli R, Marfisi RM, Carinci F, Tognoni G. Meta-analysis, clinical trials, and transferability of research results into practice. The case of cholesterollowering interventions in the secondary prevention of coronary heart disease. Arch Intern Med. 1996;156:1158-72.

5. Authors/Task Force M, Piepoli MF, Hoes AW, Agewall S, Albus C, Brotons C, Catapano AL, Cooney MT, Corra U, Cosyns B, et al. 2016 European guidelines on cardiovascular disease prevention in clinical practice: the sixth joint Task Force of the European Society of Cardiology and Other Societies on cardiovascular disease prevention in clinical practice (constituted by representatives of 10 societies and by invited experts): developed with the special contribution of the European Association for Cardiovascular Prevention \& rehabilitation (EACPR). Eur J Prev Cardiol. 2016;23:NP1-NP96.

6. Long-Term Intervention with Pravastatin in Ischaemic Disease Study G. Prevention of cardiovascular events and death with pravastatin in patients with coronary heart disease and a broad range of initial cholesterol levels. N Engl J Med. 1998;339:1349-57.

7. Randomised trial of cholesterol lowering in 4444 patients with coronary heart disease: the Scandinavian Simvastatin Survival Study (4S). Lancet. 1994; 344:1383-9.

8. Tavazzi L, Maggioni AP, Marchioli R, Barlera S, Franzosi MG, Latini R, Lucci D, Nicolosi GL, Porcu M, Tognoni G, Gissi HFI. Effect of rosuvastatin in patients with chronic heart failure (the GISSI-HF trial): a randomised, double-blind, placebo-controlled trial. Lancet. 2008;372:1231-9.

9. Kjekshus J, Apetrei E, Barrios V, Bohm M, Cleland JG, Cornel JH, Dunselman P, Fonseca C, Goudev A, Grande P, et al. Rosuvastatin in older patients with systolic heart failure. N Engl J Med. 2007;357:2248-61.

10. Buber J, Goldenberg I, Moss AJ, Wang PJ, McNitt S, Hall WJ, Eldar M, Barsheshet A, Shechter M. Reduction in life-threatening ventricular tachyarrhythmias in statin-treated patients with nonischemic cardiomyopathy enrolled in the MADIT-CRT (multicenter automatic defibrillator implantation trial with cardiac resynchronization therapy). J Am Coll Cardiol. 2012;60:749-55.
11. De Sutter J, Firsovaite V, Tavernier R. Prevention of sudden death in patients with coronary artery disease: do lipid-lowering drugs play a role? Prev Cardiol. 2002;5:177-82

12. Rahimi K, Majoni W, Merhi A, Emberson J. Effect of statins on ventricular tachyarrhythmia, cardiac arrest, and sudden cardiac death: a meta-analysis of published and unpublished evidence from randomized trials. Eur Heart J. 2012;33:1571-81.

13. Wanahita N, Chen J, Bangalore S, Shah K, Rachko M, Coleman Cl, Schweitzer $P$. The effect of statin therapy on ventricular tachyarrhythmias: a metaanalysis. Am J Ther. 2012;19:16-23.

14. Mitchell LB, Powell JL, Gillis AM, Kehl V, Hallstrom AP, Investigators A. Are lipid-lowering drugs also antiarrhythmic drugs? An analysis of the Antiarrhythmics versus implantable defibrillators (AVID) trial. J Am Coll Cardiol. 2003;42:81-7.

15. Liao YC, Hsieh YC, Hung CY, Huang JL, Lin CH, Wang KY, Wu TJ. Statin therapy reduces the risk of ventricular arrhythmias, sudden cardiac death, and mortality in heart failure patients: a nationwide population-based cohort study. Int J Cardiol. 2013;168:4805-7.

16. Chiu JH, Abdelhadi RH, Chung MK, Gurm HS, Marrouche NF, Saliba WI, Natale A, Martin DO. Effect of statin therapy on risk of ventricular arrhythmia among patients with coronary artery disease and an implantable cardioverter-defibrillator. Am J Cardiol. 2005;95:490-1.

17. Levantesi G, Scarano M, Marfisi R, Borrelli G, Rutjes AW, Silletta MG, Tognoni $G$, Marchioli R. Meta-analysis of effect of statin treatment on risk of sudden death. Am J Cardiol. 2007;100:1644-50.

18. Vyas AK, Guo H, Moss AJ, Olshansky B, McNitt SA, Hall WJ, Zareba W, Steinberg JS, Fischer A, Ruskin J, et al. Reduction in ventricular tachyarrhythmias with statins in the multicenter automatic defibrillator implantation trial (MADIT)-II. J Am Coll Cardiol. 2006;47:769-73.

19. Goldberger JJ, Subacius H, Schaechter A, Howard A, Berger R, Shalaby A, Levine J, Kadish AH, Investigators D. Effects of statin therapy on arrhythmic events and survival in patients with nonischemic dilated cardiomyopathy. J Am Coll Cardiol. 2006;48:1228-33.

20. Desai H, Aronow WS, Tsai FS, Ahn C, Lai HM, Amin H, Gandhi K, Frishman $\mathrm{WH}$, Cohen M, Sorbera C. Statins reduce appropriate cardioverterdefibrillator shocks and mortality in patients with heart failure and combined cardiac resynchronization and implantable cardioverterdefibrillator therapy. J Cardiovasc Pharmacol Ther. 2009;14:176-9.

21. Priori SG, Blomstrom-Lundqvist C, Mazzanti A, Blom N, Borggrefe M, Camm J, Elliott PM, Fitzsimons D, Hatala R, Hindricks G, et al. 2015 ESC guidelines for the management of patients with ventricular arrhythmias and the prevention of sudden cardiac death: the Task Force for the Management of Patients with ventricular arrhythmias and the prevention of sudden cardiac death of the European Society of Cardiology (ESC). Endorsed by: Association for European Paediatric and Congenital Cardiology (AEPC). Eur Heart J. 2015;36:2793-867.

22. Austin PC. An introduction to propensity score methods for reducing the effects of confounding in observational studies. Multivariate Behav Res. 2011:46:399-424.

23. Ferdinand D, Otto M, Weiss C. Get the most from your data: a propensity score model comparison on real-life data. Int J Gen Med. 2016;9:123-31.

24. Vrtovec B, Okrajsek R, Golicnik A, Ferjan M, Starc V, Schlegel TT, Radovancevic B. Atorvastatin therapy may reduce the incidence of sudden cardiac death in patients with advanced chronic heart failure. J Card Fail. 2008;14:140-4.

25. Beri A, Contractor T, Khasnis A, Thakur R. Statins and the reduction of sudden cardiac death: antiarrhythmic or anti-ischemic effect? Am J Cardiovasc Drugs. 2010;10:155-64.

26. Violi F, Calvieri C, Ferro D, Pignatelli P. Statins as antithrombotic drugs. Circulation. 2013;127:251-7.

27. Undas A, Brummel-Ziedins KE, Mann KG. Statins and blood coagulation. Arterioscler Thromb Vasc Biol. 2005:25:287-94.

28. Tekten T, Ceyhan C, Ercan E, Onbasili AO, Turkoglu C. The effect of atorvastatin on platelet function in patients with coronary artery disease. Acta Cardiol. 2004;59:311-5.

29. Lewek J, Kaczmarek K, Cygankiewicz I, Wranicz JK, Ptaszynski P. Inflammation and arrhythmias: potential mechanisms and clinical implications. Expert Rev Cardiovasc Ther. 2014;12:1077-85.

30. Albert MA, Danielson E, Rifai N, Ridker PM, Investigators P. Effect of statin therapy on C-reactive protein levels: the pravastatin inflammation/CRP evaluation (PRINCE): a randomized trial and cohort study. JAMA. 2001;286:64-70. 
31. Biasucci LM, Giubilato G, Biondi-Zoccai G, Sanna T, Liuzzo G, Piro M, De Martino $\mathrm{G}$, lerardi C, dello Russo A, Pelargonio $\mathrm{G}$, et al. C reactive protein is associated with malignant ventricular arrhythmias in patients with ischaemia with implantable cardioverter-defibrillator. Heart. 2006:92:1147-8.

32. Abuissa H, O'Keefe $\mathrm{HH}$, Bybee KA. Statins as anti-arrhythmics: a systematic review part II: effects on risk of ventricular arrhythmias. Clin Cardiol. 2009;32: 549-52.

33. Jin H, Welzig CM, Aronovitz M, Noubary F, Blanton R, Wang B, Rajab M, Albano A, Link MS, Noujaim SF, et al. QRS/T-wave and calcium alternans in a type I diabetic mouse model for spontaneous postmyocardial infarction ventricular tachycardia: a mechanism for the antiarrhythmic effect of statins. Heart Rhythm. 2017;14:1406-16.

34. De Sutter J, Tavernier R, De Buyzere M, Jordaens L, De Backer G. Lipid lowering drugs and recurrences of life-threatening ventricular arrhythmias in high-risk patients. J Am Coll Cardiol. 2000;36:766-72.

Ready to submit your research? Choose BMC and benefit from:

- fast, convenient online submission

- thorough peer review by experienced researchers in your field

- rapid publication on acceptance

- support for research data, including large and complex data types

- gold Open Access which fosters wider collaboration and increased citations

- maximum visibility for your research: over $100 \mathrm{M}$ website views per year

At BMC, research is always in progress.

Learn more biomedcentral.com/submissions 